\title{
On the França and Hama Analysis of Elastic Hadron Scattering
}

\author{
M. J. Menon \\ Instituto de Física Gleb Wataghin \\ Universidade Estadual de Campinas, UNICAMP \\ 13083-970 Campinas, SP, Brazil
}

Received on 29 September, 2006

\begin{abstract}
After reviewing the main aspects of the model-independent analysis on elastic proton-proton scattering, developed by França and Hama in the seventies, we argue that the work can be considered a paradigm for empirical analysis on elastic hadron interactions (the inverse problem). We discuss some further developments, consequences and results that have been recently obtained on the subject and which have been based on the original strategy by França and Hama.
\end{abstract}

Keywords: Elastic scattering; Hadron-induced high-energy interactions

\section{INTRODUCTION}

Elastic hadron-hadron scattering has always been a topical problem in Particle Physics. As a soft scattering state it can not be treated by standard QCD methodology in both perturbative and non-perturbative contexts. In the former case the increase of the coupling at large distances (small momentum transfer) forbids perturbative techniques and in the latter case, lattice QCD or other non-perturbative approaches, are still not able to treat soft scattering without local model inputs, that is on a pure QCD formalism. In the phenomenological context, particular data sets can be described by a large variety of models, but we do not have a widely accepted global approach able to describe all the available data, with truthful connections or basis on microscopic concepts: the underlying field theory of hadronic interactions.

In this context, model independent analysis play a crucial role in the investigation of connections between experimental data and first principles and theorems in Field Theory, providing useful information for the development of adequate calculational schemes. Lorentz invariance, unitarity, analyticity and crossing constitute the pillars of a field theory for scattering states and in this context the impact parameter and eikonal pictures, directly connected with unitarity, are a natural arena for unitarized phenomenology. Therefore, the inverse approach, aimed to extract model-independent information on the eikonal and profile functions certainly play a fundamental role in hadron interactions.

In this workshop, celebrating the 70th anniversary of Professor Yogiro Hama, we think it is important to recall, mainly for the "new generation", some fundamental results he obtained, together with Professor Humberto França, in the area of elastic hadron scattering, more than 30 years ago [1]. Also important is to recall some consequences of this work and some recent developments, which were based on their results and strategies.

To this end, in Sec. II we first contextualize the paper [1] in the late seventies and then review some topical aspects and results present there. In Sec. III we discuss some consequences of that publication and further developments that have been obtained by our group. The conclusions and some final remarks are the contents of Sec. IV.

\section{THE FRANÇA AND HAMA ANALYSIS}

\section{A. Impact parameter and eikonal pictures}

The most important physical quantity that characterizes the elastic scattering is the differential cross section:

$$
\frac{d \sigma}{d q^{2}}=\pi\left|\operatorname{Re} F\left(s, q^{2}\right)+i \operatorname{Im} F\left(s, q^{2}\right)\right|^{2},
$$

where $F$ is the scattering amplitude, $s$ and $q^{2}=-t$ the Mandelstam variables. In the impact parameter picture this amplitude is expressed as the Fourier transform of the Profile function $\Gamma$ (Fraunhofer diffraction) [2]

$$
F(s, q)=\mathrm{i} \int_{0}^{\infty} b d b J_{0}(q b) \Gamma(s, b)
$$

where $b$ is the impact parameter and $J_{0}$ is the zero order Bessel function (azimuthal symmetry assumed). In the eikonal representation the profile is given in terms of the the eikonal function $\chi$ by

$$
\Gamma(s, b)=1-e^{\mathrm{i} \chi(s, b)}
$$

and unitarity allows to connect the profile and inelastic overlap function, $G_{\text {inel }}[2]$ :

$$
2 \operatorname{Re} \Gamma(\mathrm{s}, \mathrm{b})=|\Gamma(\mathrm{s}, \mathrm{b})|^{2}+\mathrm{G}_{\mathrm{in}}(\mathrm{s}, \mathrm{b}) .
$$

This formalism can be used in two directions: (1) in eikonal models, from a suitable input for $\chi(s, b)$ and through (3), (2) and (1), the model predictions can be tested with the experimental data and some precursors are Glauber, Byers-Yang, Chou-Yang; (2) in model-independent analysis (the inverse problem), from suitable parametrization for the scattering amplitude and fit to the differential cross section data, Eq. (1), the profile (2), eikonal (3) and overlap function (4) can be extracted and some precursors are Franco, Lombard-Wilkin and França-Hama. 


\section{B. Historical context}

In the seventies the eikonal models where characterized by two competing ideas, which can be pedagogically discussed from the following suitble expression for the eikonal [3]:

$$
\operatorname{Im} \chi(s, b)=f(s) g\left(\frac{b}{R}\right),
$$

where $R$ has dimensions of lenght. On the one hand, Factorizing Eikonal models [4] assumed $R=$ constant, so that

$$
\operatorname{Im} \chi(s, b)=f(s) g(b) .
$$

On the other hand, Geometrical Scaling models [5] assumed $f(s)=$ constant and $R$ related to the total cross section by $R=$ $R(s) \propto \sqrt{\sigma_{t o t}(s)} ;$ in this case,

$$
\operatorname{Im} \chi(s, b)=g\left(\frac{b}{\sqrt{\sigma_{t o t}(s)}}\right)
$$

which implies in an uniform expansion of $\chi(s, b)$. It should be stressed that both competing approaches were able to describe satisfactorily the experimental data available at that time.

In November 1978, França and Hama submitted a novel model-independent analysis on elastic $p p$ interaction [1], which, for the first time, indicated the breakdown of the factorizing hypothesis, suggesting also the violation of the geometrical scaling [1].

\section{Fitting and data}

In their work, the imaginary part of the scattering amplitude where parametrized as a sum of exponentials in $q^{2}$ :

$$
\operatorname{Im} F(s, q)=\operatorname{Im} F(s, 0) \sum_{i=1}^{n} \alpha_{i} e^{-\beta_{i} q^{2}}
$$

The omission of the real part contribution could be explained by dispersion relations and the small value of the ratio between real and imaginary parts in the forward direction.

As commented before, in the inverse approach, the point is to fit the experimental data by means of parametrization (8) and Eq. (1), and then to extract the profile and eikonal function. With assumption of purelly imaginary amplitude, it is the imaginary part of the eikonal that can be extracted. In what follows, we shall use the same symbol $\chi$ in both cases.

A first important aspect of the analysis is the carefull compilation of the experimental data then available on protonproton differential cross sections. That was a difficult task mainly due to normalized and unnormalized data, as discussed in detail in Sect. II of [1]. The selection and compilation provided 6 sets of data at the energies $\sqrt{s}=19.5,23,30.7,44.9$, 53 and $62 \mathrm{GeV}$. A second aspect concerns the fit procedure, which, at that time, were performed without any computer code, like MINUIT or other code, but by direct determination of exponential contributions in the plot and in different intervals of momentum transfer [6]. With this procedure a good description of all data was obtained with 6 exponential factors in parametrization (8) and therefore 13 free parameters at each energy (see Table I in [1]).

\section{Results and "advices"}

By inverting Eqs. (2) and (3) the profile and eikonal were then extracted in a model-independent way. A plot of the eikonal as a function of the energy at 6 different values of the impact parameter, provided the following fundamental empirical result:

$$
\chi(s, b)=\chi_{f}(b)+\ln \left(s / s_{0}\right) \chi_{0}(b) .
$$

Therefore, the immediate conclusion is the breakdown of the factorization hypothesis. Moreover, as shown in Fig, 1 (extracted from [1]) the obtained eikonal and profile, as function of the impact parameter at different energies, indicated a nonuniform expansion, suggesting the violation of the geometrical scaling.

This focus on the eikonal, instead of the overlap function is a fundamental aspect of the approach, as clearly explained by the authors (our emphasis in what follows): "In some earlier works ${ }^{18,23}$ a peripheral increase of the total cross section has been pointed out. In all of them, however, the discussion goes around the overlap function and does not refer to the eikonal. It is clear that even when the energy variation of the eikonal is central, the one corresponding to the overlap function may appear peripheral. Thus, the ambiguity should be removed by discussing directly the opacity, if one intends to consider the internal structure of the interacting hadrons."

In the conclusions of the paper, the authors explicitly stress the violation of factorization and call the attention to the nonuniform increase of the eikonal (our emphasis): "In short, while the factorization hypothesis for the eikonal is definitely discarded, provided the assumptions introduced in Sec. III are valid, the soundness of the geometrical scaling hypothesis remains inconclusive, although there are indications of its breakdown".

\section{FURTHER RESULTS AND CONSEQUENCES}

Certainly the conclusions quoted above, based on a precise model-independent analysis, represented a fruitful and important advance in the phenomenological context. However, as exemplified in what follows, the paper did not receive the due attention for a long time.

\section{A. Some further results}

It may be useful to comment, at least, two further interesting publications on the subject. Despite the clear advice 


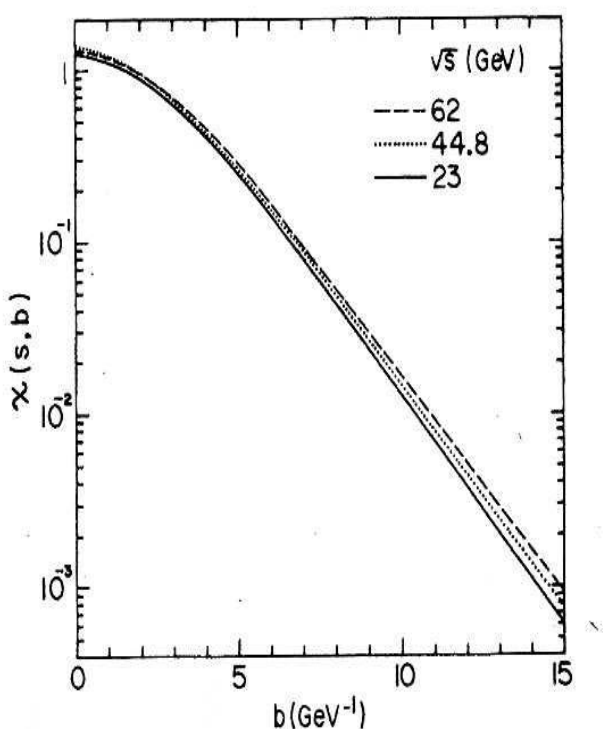

FIG. 9. The opacity, as a function of the impact parameter, for three ISR energies.

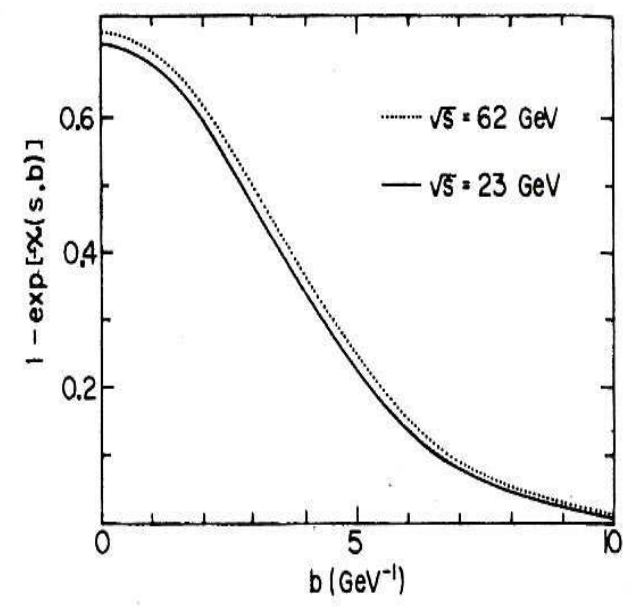

FIG. 10. The Fourier transform of the amplitude for the two extreme ISR energies.

FIG. 1: Results for the eikonal (left) and the profile function from the model-independent analysis by França and Hama. Figures extracted from [1].

on the importance of the eikonal information, one year latter, Amaldi and Schubert put efforts on the extraction of the overlap function, concluding that the geometrical scaling hypothesis was valid at the ISR energy region $(23-62 \mathrm{GeV})$ : "This impact parameter analysis has smaller errors than any other previously made, and confirms the good agreement with the geometrical scaling model..." [3]. The analysis by Amaldi and Schubert also treated the factorizing hypothesis and led to the same conclusion previously reached by França and Hama, but without reference to their publication. As it is well known, the scaling hypothesis was definitely discarded in 1984 when it was discovered the increase in the ratio between elastic and total cross section from $\approx 0.17$ at the ISR to $\approx 0.22$ at the $S \bar{p} p S$ [7] and that was the end of the geometrical scaling in the elastic sector.

It may also be interesting to note that 15 years after [1] it was "introduced" by Hüfner, Povh and Wälde a phenomenological parametrization for the eikonal in the form [8]

$$
\chi(s, b)=\chi_{0}(b)+\eta \ln \left(s / s_{0}\right) \chi_{1}(b),
$$

which is the same model-independent result by França-Hama. Once more, unfortunately there was no reference to their previous result.

\section{B. Some consequences}

During the eighties and also nineties new experimental data provided useful information on several aspects of the elastic hadronic interactions. Among these, data taken from $p p$ scattering at $\sqrt{s}=27.5 \mathrm{GeV}$ in the region $3.5 \leq q^{2} \leq 14 \mathrm{GeV}^{2}$ [9] indicated the constance of the slope of the differential cross section in the region of large momentum transfer (above $\approx 4$ $\mathrm{GeV}$ ), as illustrated in Fig. 2. Moreover, data from the $S \bar{p} p S$ allowed the investigation of $\bar{p} p$ scattering up to $1.8 \mathrm{TeV}$ and have shown that the contribution of the forward real part could reach $\approx 13 \%$.

In the late nineties, based on these novel information and inspired by the empirical approach developed by França-Hama, we started the investigation of the inverse problem in elastic scattering. In a first step we treated only $p p$ scattering for which we introduced the following complex parametrization [10]

$$
F\left(s, q^{2}\right)=\frac{\rho(s)}{\alpha_{1}+\alpha_{2}} \sum_{j=1}^{n} \alpha_{j} \sum_{j=1}^{2} \alpha_{j} e^{-\beta_{j} q^{2}}+\mathrm{i} \sum_{j=1}^{n} \alpha_{j} e^{-\beta_{j} q^{2}}
$$

where $\alpha_{j}, \beta_{j}(j=1,2, \ldots, n)$ are real free parameters and $\rho(s)$ is the value of the forward ratio between real and imaginary parts of the amplitude, extracted from the experiments at each energy. Based on the constance of the differential cross section at large momentum transfer (Fig. 2), we added to each ISR set the data at $27.5 \mathrm{GeV}$ and fitted each set through the 


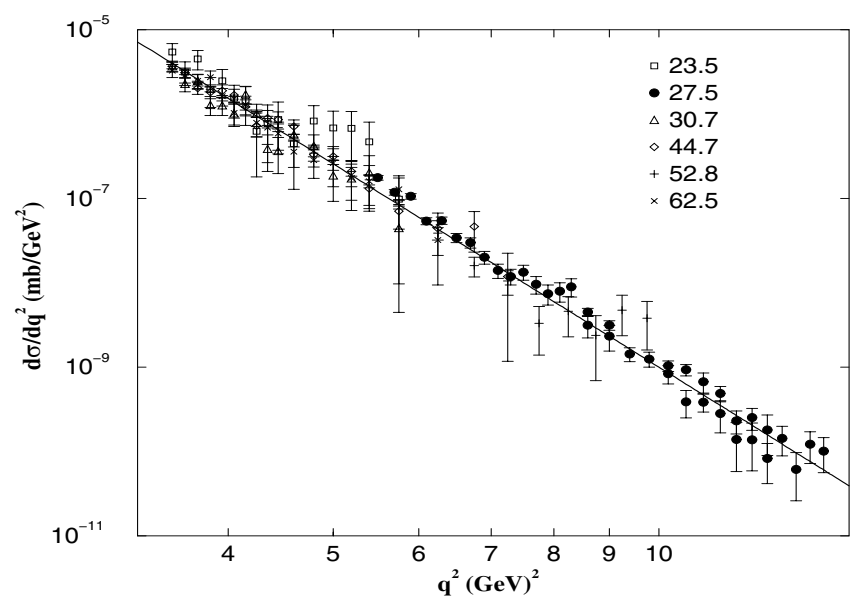

FIG. 2: Differential cross section data on $p p$ scattering in the region of large momentum transfer. The curve represents a fit with one exponential term.

CERN-MINUIT code. A typical fitting is illustrated in Fig. 3. By means of a semi-analytical method [10] we were able to extract information on the eikonal in the momentum transfer space showing the existence of a zero (change of signal) at $q^{2}=7 \pm 2 \mathrm{Gev}^{2}[10]$.

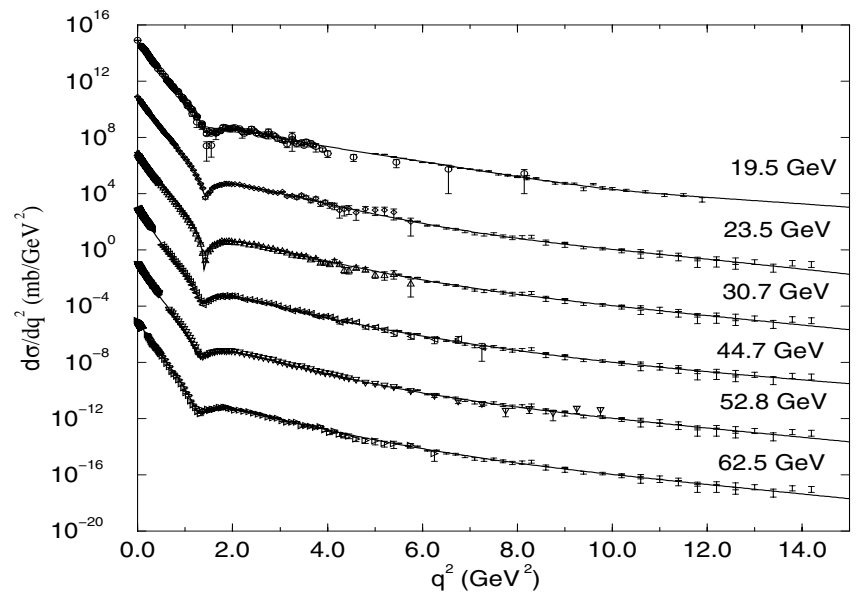

FIG. 3: Description of the $p p$ elastic scattering by means of parametrization (11) [10].
After that, we extended the analysis to $\bar{p} p$ scattering [11] and in all these results the uncertainties from the fit parameters have been propagated to the impact parameter and momentum transfer spaces. In [11] we also present a critical review, with detailed discussions, on model-independent analysis of elastic hadron scattering.

These analysis have been constantly improved and led, recently, to an almost model-independent approach, where the dependence of each free fit parameter with the energy is inferred on empirical basis [12].

\section{CONCLUSIONS AND FINAL REMARKS}

We have presented a short review on the seminal paper by França-Hama on elastic scattering. We discussed the importance and implication of the results not only in the context of the seventies but also recently.

I would like to invite the reader, in special the "new generation", to consult the original manuscript, paying attention on some particular aspects such as the careful compilation and discussion on the experimental data then available, the accurate analysis, the precision of the information extracted, the conditions then available and in the exact solution and physical interpretation given by the authors. In this sense I think this paper is a paradigm for the inverse problem in elastic scattering.

But that is not all. There are lots of things to learn from this special paper and I refer not only to the physics quoted above. It also shows that not even the community is prepared or interested to understand and/or give the adequate respect to a novel result.

Acknowledgments. It is a pleasure to congratulate Professor Yogiro Hama for his 70th anniversary and to express my deeply thankfull for the seeds launched and also for all the support to our group at Unicamp. Financial support by FAPESP (Contract No.04/10619-9).
[1] H. M. França and Y. Hama, Phys. Rev. D 19, 3261 (1979).

[2] V. Barone, E, Predazzi, High-Energy Particle Diffraction (Spring-Verlag, Berlin, 2002).

[3] U. Amaldi, K. R. Schubert, Nucl. Phys. B 166, 301 (1980).

[4] H. Cheng, J. K. Walker, and T. T. Wu, Phys. Lett. B 44, 97 (1973); F. Hayot and U. P. Sukhatme, Phys. Rev. D 10, 2183 (1974).

[5] J. Dias de Deus, Nucl. Phys. B 59, 231 (1973); A. J. Buras and J. Dias de Deus, Nucl. Phys. B 71, 481 (1974).

[6] Y. Hama, private communication.
[7] M. Bozzo et al. Phys. Lett. B 147, 392 (1984).

[8] J. Hüfner, B. Povh, and E. Wälde, Z. Phys. C 63, 631 (1994).

[9] W. Faissler et al, Phys. Rev. D 23, 33 (1981).

[10] P. A. S. Carvalho, M. J. Menon, Phys. Rev. D 56, 7321 (1997).

[11] P. A. S. Carvalho, A. F. Martini, and M. J. Menon, Eur. Phys. J. C 39, 359 (2005).

[12] R. F. Ávila, S. D. Campos, M. J. Menon, and J. Montanha, Eur. Phys. J. C 47, 171 (2006). 